\title{
Correlation between Cognition and Function across the Spectrum of Alzheimer's Disease
}

\author{
H. Liu-Seifert ${ }^{1}$, E. Siemers ${ }^{1}$, K. Selzler ${ }^{1}$, K. Sundell 1 , P. Aisen ${ }^{3}$, J. Cummings ${ }^{4}$, J. Raskin ${ }^{2}$, R. Mohs ${ }^{1 *}$ for the \\ Alzheimer's Disease Neuroimaging Initiative
}

\begin{abstract}
1. Lilly Research Laboratories, Lilly Corporate Center, Indianapolis, IN, USA; 2. Eli Lilly Canada, Toronto, Canada; 3. University of Southern California, Alzheimer's Therapeutic Research Institute, San Diego, CA, USA; 4. Cleveland Clinic Lou Ruvo Center for Brain Health, Las Vegas, NV, USA

${ }^{*}$ Retired employee of Eli Lilly and Company

Corresponding Author: Hong Liu-Seifert, Eli Lilly and Company, Lilly Corporate Center, Indianapolis, IN 46285, Liu-seifert_hong@lilly.com, +1 317-433-0662
\end{abstract}

\begin{abstract}
BACKGROUND: Both cognitive and functional deterioration are characteristic of the clinical progression of Alzheimer's disease (AD).

OBJECTIVES: To systematically assess correlations between widely used measures of cognition and function across the spectrum of AD.

DESIGN: Spearman rank correlations were calculated for cognitive and functional measures across datasets from various AD patient populations.

SETTING: Post-hoc analysis from existing databases.

PARTICIPANTS: Pooled data from placebo-treated patients with mild (MMSE score $\geq 20$ and $\leq 26$ ) and moderate (MMSE score $\geq 16$ and $\leq 19$ ) AD dementia from two Phase 3 solanezumab (EXPEDITION/2) and two semagecesatat (IDENTITY/2) studies and normal, late mild cognitive impairment (LMCI) and mild AD patients from the Alzheimer's Disease Neuroimaging Initiative 2-Grand Opportunity (ADNI-2/GO).

INTERVENTION (IF ANY): Placebo (EXPEDITION/2 and IDENTITY / 2 subjects).

MEASUREMENTS: Cognitive and functional abilities were measured in all datasets. Data were collected at baseline and every three months for 18 months in EXPEDITION and IDENTITY studies; and at baseline, 6, 12, and 24 months in the ADNI dataset.

RESULTS: The relationship of cognition and function became stronger over time as AD patients progressed from preclinical to moderate dementia disease stages, with the magnitude of correlations dependent on disease stage and the complexity of functional task. The correlations were minimal in the normal control population, but became stronger with disease progression.

CONCLUSIONS: This analysis found that measures of cognition and function become more strongly correlated with disease progression from preclinical to moderate dementia across multiple datasets. These findings improve the understanding of the relationship between cognitive and functional clinical measures during the course of AD progression and how cognition and function measures relate to each other in AD clinical trials.
\end{abstract}

Key words: Cognition, function, correlation, Alzheimer's disease.

\section{Introduction}

$\Lambda$ lzheimer's disease $(\mathrm{AD})$ is characterized by the relentless deterioration of both cognition and function. The clinical symptoms of the earliest stages of $\mathrm{AD}$ begin with cognitive decline, typically involving episodic memory, with apparent effects on function occurring later in the disease progression. This sequence in the clinical manifestations of the disease is reflected by current clinical diagnostic criteria across the spectrum of $\mathrm{AD}(1,2)$; overt functional impairment is not included in the criteria before the dementia stage. Both cognition and function are highly valued by patients and caregivers, and recent studies suggest that cognitive decline is meaningful, even before it adversely affects function (3).

For currently approved symptomatic treatments used in patients with mild, moderate or severe dementia, changes in cognition and global or functional measures were required as co-primary measures to assess effect of treatments on core cognitive deficits (as measured by the ADAS-Cog) as well as the clinical meaningfulness of a drug effect as assessed by a degree of global or functional outcomes (4). However, many current treatments in development target the underlying disease process with intent to slow disease progression. In addition, there is a growing consensus that these treatments may have greater benefit when started earlier in the disease continuum $(5,6)$, when there is often little to no measureable functional deficit and symptoms can be measured only by cognitive tests (1). Additionally, recent analyses have investigated the relationship between cognition and function and shown a temporal lag between cognitive changes and functional changes $(7,8)$. Taken together, these analyses suggest that the historical paradigm of co-primary endpoints developed for symptomatic agents expected to improve cognition and function within a relatively short time period in later stages of the disease may not be optimal for clinical trials of putative disease-modifying treatments, aiming to slow 
disease progression in earlier stages of the disease.

Limited literature to date has directly compared the associations between cognition and function across the spectrum of AD severity. A few studies have found that executive function predicts functional behaviors, as measured by the AD Cooperative Study-Activities of Daily Living instrumental scale (ADCS-iADL) in both normal populations (9-11) as well as in AD (1214). Similarly, memory has also been shown to predict functional ability in normal elderly populations (15), AD (16), and mild cognitive impairment (MCI) (17). A similar study using longitudinal data determined there was a strong association between cognition, including memory, with subsequent physical functional abilities (18).

In recent studies, we and others have investigated the relationship between cognition and function across the course of $\mathrm{AD}$ using autoregressive cross-lagged (ARCL) panel analyses and path analyses $(7,8,19)$. ARCL panel analysis is a classical structural equation model and is designed to assess the strength of potential reciprocal causal relationships between outcomes and explore inference of influence of one variable over another. The findings from ARCL analyses have demonstrated cognitive decline precedes and predicts functional decline in the natural course of $\mathrm{AD}$, but function was unable to predict subsequent changes in cognition ( 7 , 8). Additionally, path analyses were used to quantify the relationship between treatment effects on variables, such as measures of cognition and function. Based on secondary analyses of patients with mild dementia in the EXPEDITION and EXPEDITION2 studies, the treatment effect on function was mediated primarily by the treatment effect on cognition (19). While the ARCL analyses measured the temporal delay between cognition and function over time, it is unclear how the two measures are correlated with each other at any given time point.

With few studies exploring the cross-sectional relationship between cognition and function at various time points during the stages of $\mathrm{AD}$, we conducted this post-hoc analysis to systematically assess correlations between cognition and function from preclinical to moderate dementia disease stages, over multiple time points. Recent publications have assessed the qualitative (temporal) relationship between cognition and function, mainly in the mild AD stage. This study expands the scope to a broader spectrum of $\mathrm{AD}$, from normal cognition to moderate AD, and includes more quantitative assessments of the cognitive-functional relationship using correlations. Understanding how the magnitudes of the correlations change over time may be useful in $\mathrm{AD}$ clinical trial designs and may help physicians provide treatment expectations for patients and caregivers.

\section{Methods}

\section{Subjects}

Data from placebo patients with mild (baseline Mini-Mental Status Examination [MMSE] score $\geq 20$ and $\leq 26$ ) to moderate (baseline MMSE score $\geq 16$ and $\leq 19)$ AD dementia were pooled from two Phase 3 solanezumab studies (EXPEDITION and EXPEDITION2) and two Phase 3 semagacestat studies (IDENTITY and IDENTITY2), separately. Data obtained from the Alzheimer's Disease Neuroimaging Initiative (ADNI-2/ GO) databases (20) (adni.loni.usc.edu) included normal, late mild cognitive impairment (LMCI), and mild AD dementia subjects. Additional information regarding the three study programs as well as the outcome measures used in this post-hoc analysis, are provided below.

\section{EXPEDITION Study Program}

EXPEDITION and EXPEDITION2 were multicenter, double-blind, Phase 3 studies of solanezumab. Solanezumab is a humanized monoclonal antibody designed to increase clearance of soluble amyloid- $\beta$ $(\mathrm{A} \beta)$ from the brain and is currently being studied as a potential disease-modifying agent for the treatment of AD. All patients who participated in the EXPEDITION study program provided informed consent and the study protocols were approved by ethical review boards. The primary outcomes of the solanezumab clinical trials have been reported previously (21).

\section{IDENTITY Study Program}

IDENTITY and IDENTITY2 were multicenter, doubleblind, Phase 3 semagacestat studies. Semagacestat is a gamma secretase inhibitor that was studied as a putative disease-modifying agent for the treatment of AD. Treatment in both studies was terminated prematurely based on data that showed cognitive worsening in patients treated with semagacestat compared to placebo. Patients were then followed for seven months to collect additional safety data. All patients enrolled in the study provided informed consent before participation and the study protocols were approved by ethical review boards. The results from the IDENTITY studies have been reported previously (22).

\section{ADNI Program}

A third dataset used in this analysis was obtained from the AD Neuroimaging Initiative (ADNI) database (20) adni.loni.usc.edu. The ADNI was launched in 2003 as a public-private partnership, led by Principal Investigator Michael W. Weiner, MD. The primary goal of ADNI has 
been to test whether serial magnetic resonance imaging (MRI), positron emission tomography (PET), other biological markers, and clinical and neuropsychological assessment can be combined to measure the progression of $\mathrm{MCI}$ and early AD. For up-to-date information, see (23) www.adni-info.org.

\section{Outcome Measures - EXPEDITION $\mathcal{E}$ IDENTITY Programs}

In the EXPEDITION and IDENTITY studies, cognitive and functional outcome measures were assessed at baseline and at six post-baseline time points every 3 months for 18 months. Cognitive ability was assessed using the 14-item AD Assessment Scale-Cognitive subscale (ADAS-Cog 14 ) with a score range of 0 to 90 (with higher scores indicating greater disability) (24). Function was measured with the AD Cooperative StudyActivities of Daily Living Scale, which includes an instrumental subscale (ADCS-iADL) and basic subscale (ADCS-bADL). The iADL is comprised of items 7 through 23 of the ADCS-ADL scale, with scores from 0 to 56 (lower score denoting greater functional loss) and was designed to measure higher level functional activities such as managing finances and preparing meals. The bADL scores range from 0 to 22 and measures more rudimentary activities such as feeding and bathing (25).

\section{Outcome Measures - ADNI}

In the ADNI dataset, cognition was measured using the 11-item AD Assessment Scale-Cognitive subscale (ADAS- $\operatorname{Cog}_{11}$ ) with a score range of 0 to 70 (with higher scores indicating greater disability). Functional ability was measured using the Functional Assessment Questionnaire (FAQ) (26) with scores ranging from 0 to 30 (higher score representing more impairment, which is opposite from the ADCS-ADL).

\section{Statistical Analyses}

Spearman rank correlations between actual scores for cognition and function were calculated at each time point collected across all three datasets and in the various $\mathrm{AD}$ subject populations.

Additional analyses were performed in the pooled mild AD population from the EXPEDITION and EXPEDITION2 studies (21) to further examine the association between cognitive and functional progression (IDENTITY / 2 and ADNI-2/GO were not included in these analyses due to the limited amount of data as a result of early termination and smaller sample sizes, respectively). First, the frequency of the magnitude of change in cognitive scores, as measured by ADAS$\operatorname{Cog}_{14}$, was established and categorized as one of the following four categories for cognition as defined by
Vellas and colleagues using ADAS-Cog $\operatorname{Cog}_{11}$ at 18 months (27): improvement (improvement of 3 or more points), stable (improvement of 2 points to decline of 2 points), moderate decline (decline of 3 to 7 points), severe decline (decline of more than 7 points). For each of these four categories of change in cognitive scores, the distribution of the changes in the functional scores, as measure by ADCS-iADL, were derived. In each of the distributions, the frequency of a given change in iADL was calculated.

\section{Results}

\section{EXPEDITION Study Results}

In mild AD subjects from the EXPEDITION studies, cognition as measured by ADAS- $\operatorname{Cog}_{14}$ was correlated more with the iADLs than with bADLs (Figure 1a). In the moderate $\mathrm{AD}$ subjects, the correlations were much more similar for iADLs and bADLs (Figure 1b). Correlations between cognition and basic function were numerically higher in the moderate AD than in the mild AD subject group.

\section{IDENTITY Study Results}

Similar to EXPEDITION, in the IDENTITY studies, cognition as measured by ADAS- $\operatorname{Cog}_{14}$ was correlated more with iADLs than bADLs in mild AD (Figure 2a). In moderate $\mathrm{AD}$ subjects, however, the correlations were comparable between iADLs and bADLs (Figure 2b).

\section{ADNI Study Results}

In the ADNI dataset, the correlations between cognition, measured by ADAS-Cog ${ }_{11}$, and function, measured by FAQ, were minimal in the normal population, greater in the LMCI subjects, and continued to increase in the mild AD subjects (Figure 3).

\section{Frequency Analyses}

In the frequency analyses, approximately $50 \%$ of the mild AD patients had a worsening of 5 points or more on ADAS- $\operatorname{Cog}_{14}$ during the 18 months of follow up (Figure 4). A greater cognitive decline was associated with a greater functional decline (Figure 5).

\section{Additional Correlations}

Correlations were calculated between changes in cognitive and changes in functional scores: the same increase in correlation strength was observed from mild to moderate $\mathrm{AD}$ (data not shown). 
Figure 1a. Correlations between cognition (ADAS-Cog 14 ) and function (ADCS-iADL [dark red] and bADL [light red]) in mild AD in the EXPEDITION studies. Data from Liu-Seifert H, et al. J Alzheimers Dis. 2015b;43(3):949-955

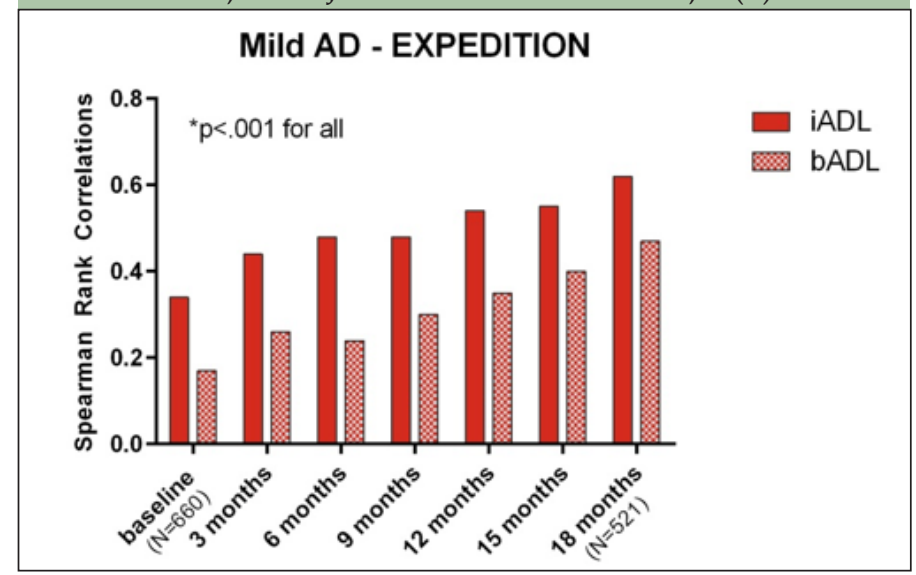

Figure 1b. Correlations between cognition (ADAS- $\operatorname{Cog}_{14}$ ) and function (ADCS-iADL [dark blue] and bADL [light blue]) in moderate AD in the EXPEDITION studies

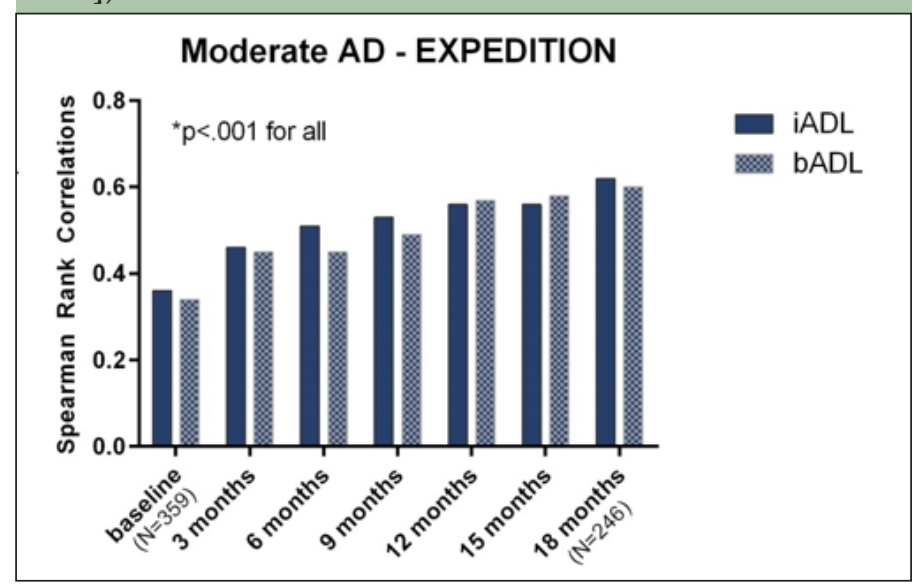

\section{Discussion}

In these analyses using multiple independent datasets, Spearman rank correlations were assessed to evaluate the relationship between cognition and function across the spectrum of the disease. The results from these analyses demonstrated that cognition and function became stronger over time as AD patients deteriorated, with correlations ranging from 0.07 to 0.62 for normal to moderate $\mathrm{AD}$ population, respectively, over time (Figure $3)$. The magnitude of the correlations also depended on the complexity of functional ability, as shown in Figures 1 and 2. This study is unique in including a broad range of disease states within the AD spectrum, from normal to moderate $\mathrm{AD}$, which has not been systematically evaluated previously. The results from this study provide further understanding regarding the association of cognition and function at any given time point and have real world applications to ongoing AD clinical trials.
Figure 2a. Correlations between cognition (ADAS-Cog 14 ) and function (ADCS-iADL [dark red] and bADL [light red]) in mild AD in the IDENTITY studies

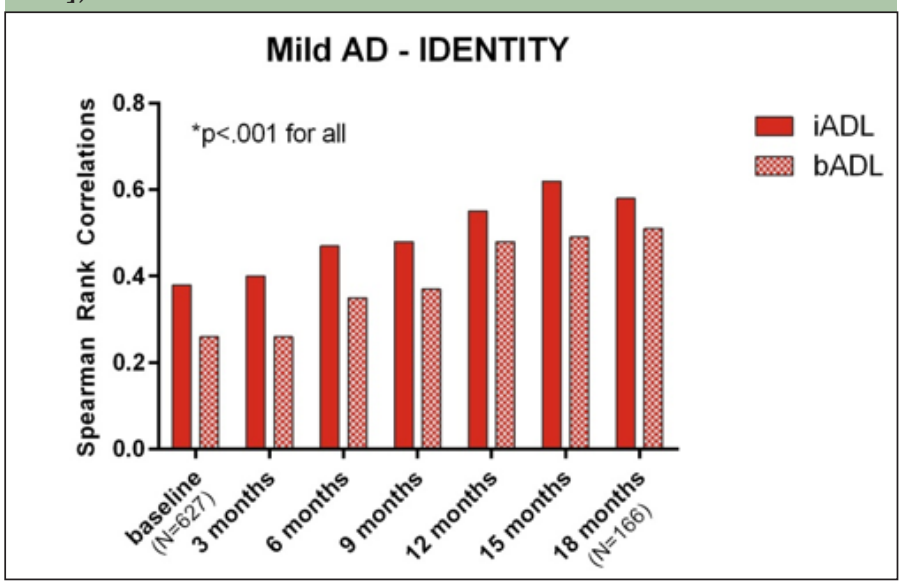

Figure $2 \mathrm{~b}$. Correlations between cognition (ADAS-Cog 14 ) and function (ADCS-iADL [dark blue] and bADL [light blue]) in moderate AD in the IDENTITY studies

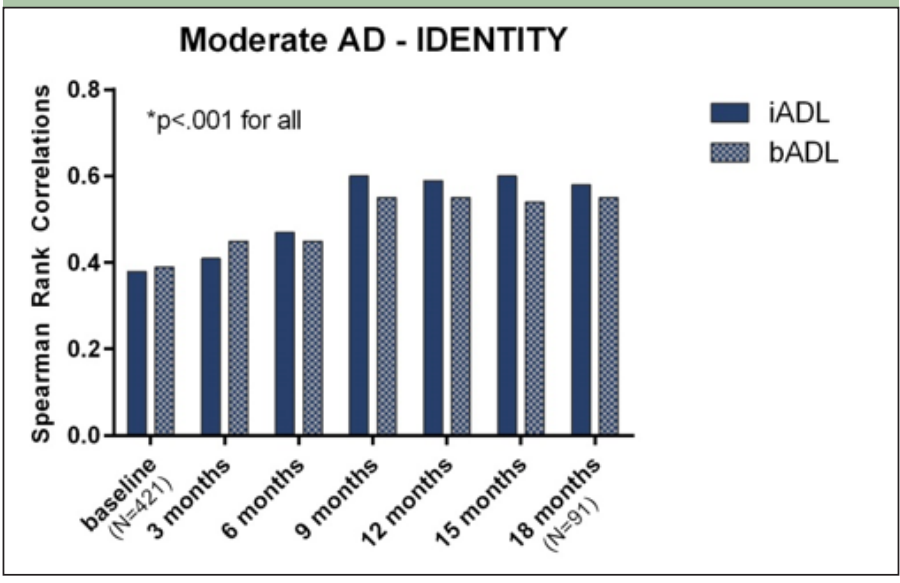

In general, there was a stronger correlation observed between cognition and the instrumental subscale of the activities of daily living compared with the basic subscale in mild $\mathrm{AD}$ subjects. These findings may reflect the early involvement or greater sensitivity of instrumental functions compared to basic functions in the evolution of $\mathrm{AD}$ dementia or a ceiling effect of the scale regarding basic functions, which are substantially less impaired in mild patients. A continual increase in the correlations between cognition and basic function as subjects progressed from mild to moderate stages of AD supports this concept as basic functions are more impaired in later disease stages.

In the ADNI dataset, the correlation between cognition and function was nominal in the normal population compared with the LMCI or mild dementia subjects, supporting the hypothesis that the correlation increases as disease progresses. In the normal population, the magnitudes of the correlations are smaller due both to the limited cognitive impairment and to the lack of the ability of the clinical measures to detect more subtle 
Figure 3. Correlations between cognition (ADAS- $\log _{11}$ ) and function (FAQ) in the normal population (left), LCMI (middle), and mild AD (right) from the ADNI dataset

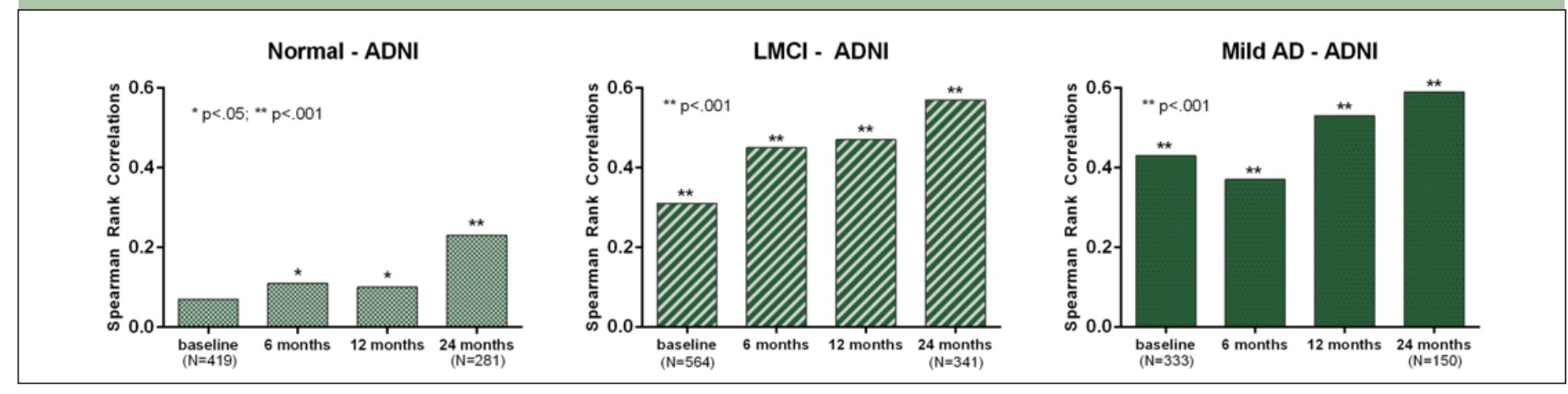

Figure 4. Frequency $(\%)$ of mild $\mathrm{AD}$ patients from EXPEDITION studies as measured by the last observation carried forward (LOCF) of cognition measured by the magnitude of change in the ADAS- $\log _{14}$ and categorized as improvement (green), stable (blue), moderate decline (orange), or severe decline (red)

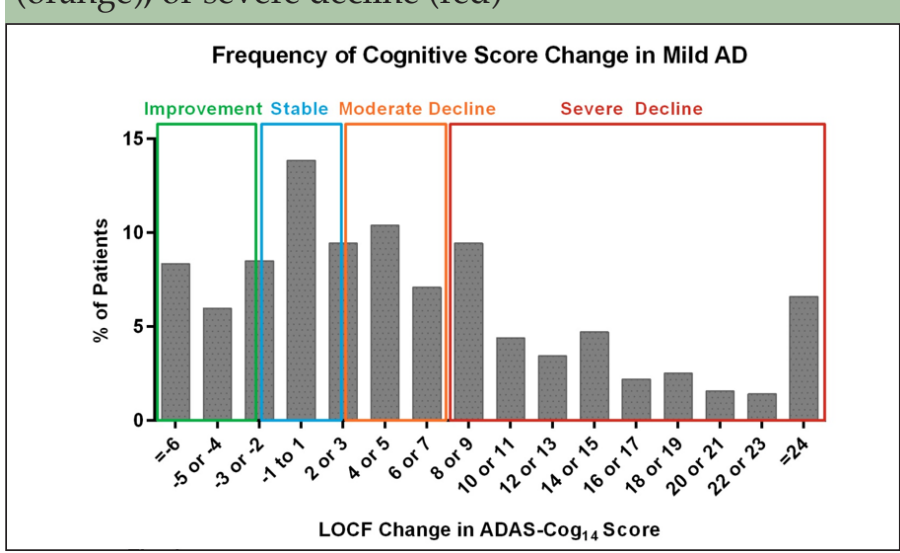

differences. Thus, across EXPEDITION, IDENTITY and ADNI datasets, the increase in correlations within the studies and across disease stages suggest that as disease progresses, cognition becomes more clearly related to function, and the two measures become more strongly associated. The magnitudes of the correlations between cognition and function are largely comparable in the mild AD population across the three independent datasets. The correlations ranged from approximately 0.4 at baseline to approximately 0.6 at the end of each study (18 months for EXPEDITION and IDENTITY datasets; 24 months for the ADNI dataset).

The frequency analyses in the EXPEDITION mild placebo-treated AD subjects showed that certain magnitudes of cognitive decline/worsening were able to predict certain magnitudes of functional decline. For example, approximately $70 \%$ of patients who had severe cognitive decline ( $\geq 8$ points in 18 months) had a functional decline of 6 points or more in instrumental daily activities. This is consistent with the findings by Vellas and colleagues (27), showing that patients with severe cognitive decline also had loss of basic function of daily activities, suggesting that cognition is a valid endpoint and can predict functional outcomes.

As our datasets did not include patients with severe dementia, this post-hoc analysis did not directly investigate the relationship between cognition and function in the severe stage of the disease. However, a previous study by Wajman and colleagues (28) determined that there was a progressive increase in correlation between specific instruments for diagnosis of severe dementia and functional scales. These findings in combination with our current analyses, suggest that cognition and function become more strongly correlated from normal aging through late stages of AD dementia.

There are limitations to these post-hoc analyses. The findings are based on existing cognitive and functional outcome measures that are commonly used in AD clinical trials; however, more sensitive measurement tools may reveal a different magnitude in correlation especially in earlier disease states. The correlations between cognition and instrumental function may also reach a plateau going from mild to moderate stages of $\mathrm{AD}$ because of the limited sensitivity of the scales. Additionally, in the EXPEDITION and IDENTITY study programs, the correlations between cognition and function at baseline may have been influenced by the study inclusion and exclusion criteria - subjects had to have specific cognitive scores (based on MMSE) to be included. There are also limitations of small sample sizes, particularly in the IDENTITY program, which was terminated early, and the ADNI dataset. Further, it is possible that there were some learning effects in the individual study populations, especially for subjects with the mildest deficits, which could have increased the magnitude of correlations at later time points. Also, ADCS-ADL data were available from the clinical trials and FAQ data from ADNI, so these tools may have different performance characteristics in the analyses. Lastly, the categories in the frequency analyses were originally determined by the ADAS- $\operatorname{Cog}_{11}$, so the categories might be slightly shifted if they were determined using a different subscale, such as the 14-item ADAS-Cog. 
Figure 5. Frequency (\%) of magnitude of change was compared in the functional scores measured by ADCS-iADL based on the categorized change in cognitive scores measured by ADAS-Cog ${ }_{14}$ in the EXPEDITION studies

Improvement

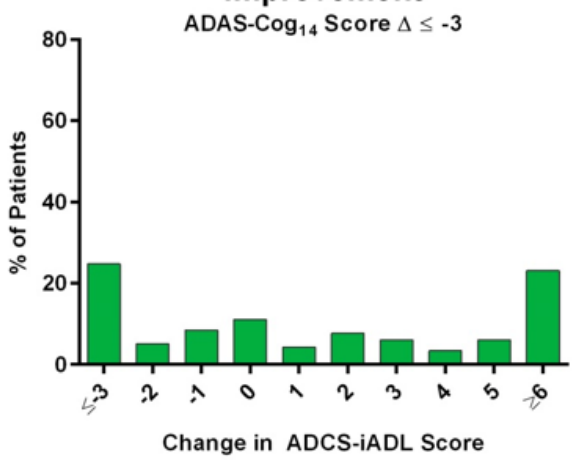

Moderate Decline

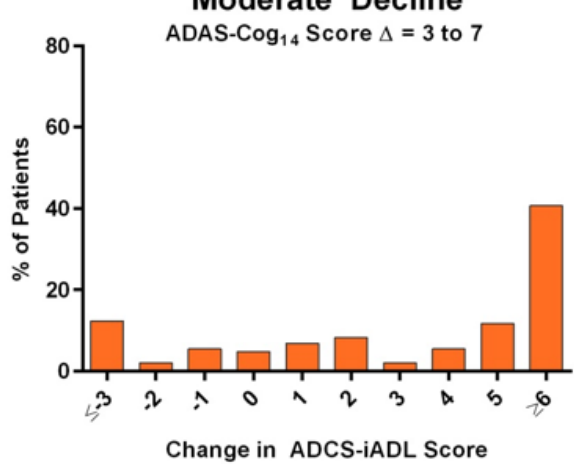

Stable

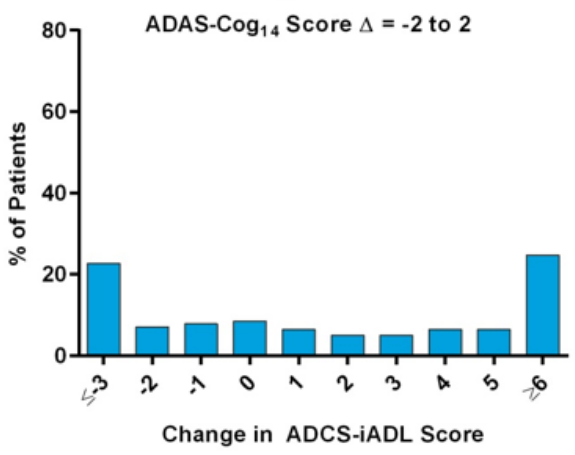

Severe Decline

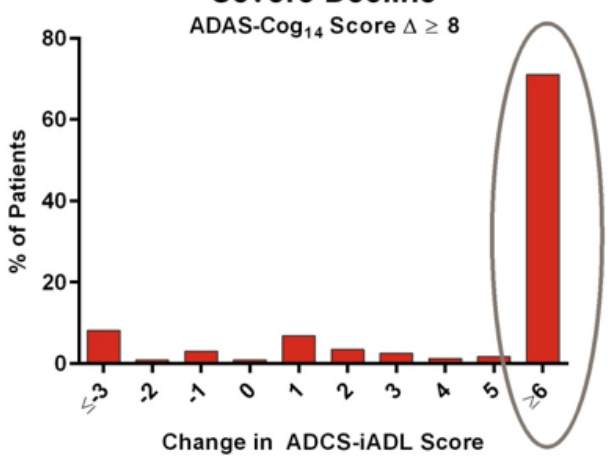

\section{Conclusions}

Cognition and function became increasingly correlated over the spectrum of the progressing severity of $\mathrm{AD}$ based on multiple independent clinical trial datasets, and the consistency of these results suggests they may be more broadly generalizable. This post-hoc analysis supports the hypothesis that instrumental functional measures are more related to cognitive abilities than basic functions, especially in the earlier stages of AD. In combination with our previous analyses, we conclude that cognitive decline may anticipate and predict functional decline in clinical trials, which might impact the use of a functional measure as a co-primary outcome with cognition. Patients with very mild AD will have no or minimal functional deficits and these will emerge as cognition declines.

The results from these analyses may help to better understand the relationship between cognitive and functional clinical measures during the entire course of $\mathrm{AD}$ progression and help foster better predictions in management of $\mathrm{AD}$ care. In particular, compared to previous publications, this post-hoc analysis expanded beyond mild $\mathrm{AD}$ to include the range of cognitively normal subjects to moderate AD patients; and also provided quantitative magnitudes of the correlations between cognition and function. Together, these findings will support future clinical trial design across the broad spectrum of the disease and may help physicians provide treatment expectations for patients and caregivers.

Acknowledgements: The authors would like to thank the Solanezumab and Semagacestat Study Groups, the patients and their caregivers for their voluntary participation, and the principal investigators and their staff. We would also like to thank ADNI for letting us analyze their data. The authors thank Drs. Ann Hake and Malgorzata Leyk for their scientific and statistical review, respectively, as well as Laura Ramsey for her editorial assistance. The data obtain from the ADNI database was funded by the Alzheimer's Disease Neuroimaging Initiative (ADNI) (National Institutes of Health Grant U01 AG024904) and DOD ADNI (Department of Defense award number W81XWH-12-2-0012). ADNI is funded by the National Institute on Aging, the National Institute of Biomedical Imaging and Bioengineering, and through generous contributions from the following: AbbVie, Alzheimer's Association; Alzheimer's Drug Discovery Foundation; Araclon Biotech; BioClinica, Inc.; Biogen; Bristol-Myers Squibb Company; CereSpir, Inc.; Eisai Inc.; Elan Pharmaceuticals, Inc.; Eli Lilly and Company; EuroImmun; F. Hoffmann-La Roche Ltd and its affiliated company Genentech, Inc.; Fujirebio; GE Healthcare; IXICO Ltd.; Janssen Alzheimer Immunotherapy Research \& Development, LLC.; Johnson \& Johnson Pharmaceutical Research and Development LLC.; Lumosity; Lundbeck; Merck \& Co., Inc.; Meso Scale Diagnostics, LLC.; NeuroRx Research; Neurotrack Technologies; Novartis Pharmaceuticals Corporation; Pfizer Inc.; Piramal Imaging; Servier; Takeda Pharmaceutical Company; and Transition Therapeutics. The Canadian Institutes of Health Research is providing funds to support ADNI clinical sites in Canada. Private sector contributions are facilitated by the Foundation for the National Institutes of Health (www.fnih.org). The grantee organization is the Northern California Institute for Research and Education, and the study is coordinated by the Alzheimer's Disease Cooperative Study at the University of California, San Diego. ADNI data are disseminated by the Laboratory for Neuro Imaging at the University of Southern California.

Funding: Eli Lilly and Company (Indianapolis, Indiana, USA) provided funding for this project and was involved in study conduct and analysis and 
preparation and approval of the final manuscript. Clinical trial registration identifiers: NCT00905372, NCT00904683, NCT00594568, NCT00762411. Part of the data used in preparation of this article was obtained from the ADNI database (adni.loni.usc.edu). As such, the investigators within the ADNI contributed to the design and implementation of ADNI and/or provided data but did not participate in analysis or writing of this report.

Conflict of interest: Drs. Liu-Seifert, Siemers, Selzler, Raskin, and Ms. Sundell are employees and minor shareowners of Eli Lilly and Company. Dr. Aisen serves on a scientific advisory board for NeuroPhage; has served as a consultant to Elan, Wyeth, Eisai, Schering-Plough, Bristol-Myers Squibb, Eli Lilly and Company, NeuroPhage, Merck, Roche, Amgen, Genentech, Abbott, Pfizer, Novartis, Bayer, Astellas, Dainippon, Biomarin, Solvay, Otsuka, Daiichi, AstraZeneca, Janssen, Medivation, Ichor, Toyama, Lundbeck, Biogen Idec, iPerian, Probiodrug, Somaxon, Biotie, Cardeus, Anavex, Kyowa Hakko Kirin Pharma, Medtronic; and receives research support from Eli Lilly and Baxter, and the NIH [NIA U01-AG10483 (PI), NIA U01-AG024904 (Coordinating Center Director), NIA R01-AG030048 (PI), and R01-AG16381 (Co-I)]. Dr. Cummings has provided consultation to Acadia, ADAMAS, Anavex, Avanir, Baxter, Boehinger-Ingelheim, Bristol-Myers Squibb, Eisai, EnVivo, Genentech, GE Healthcare, GlaxoSmithKline, Lilly, Lundbeck, Medavante, Merck, Novartis, Otsuka, Pfizer, Prana, QR Pharma, Resverlogix, Roche, Sonexa, Suven, Takeda, and Toyoma. Dr. Mohs is a minor shareholder and a retired employee of Eli Lilly and Company.

Ethical standards: Ethical review board approval and informed consent of subjects were reported in the primary publications of the studies noted in this article.

\section{References}

1. Albert MS, DeKosky ST, Dickson D, et al. The diagnosis of mild cognitive impairment due to Alzheimer's disease: Recommendations from the National Institute on Aging-Alzheimer's Association workgroups on diagnostic guidelines for Alzheimer's disease. Alzheimer Dement 2011;7:270-279.

2. McKhann GM, Knopman DS, Chertkow H, et al. The diagnosis of dementia due to Alzheimer's disease: recommendations from the National Institute on Aging-Alzheimer's Association workgroups on diagnostic guidelines for Alzheimer's disease. Alzheimer Dement 2011;7:263-269.

3. Ropacki D, Hannesdottir K, Hendrix S, et al. Consortia driven approach to addressing clinical meaningfulness in early AD. Alzheimer Dement 2014;10:P137.

4. Katz R. FDA: Evidentiary Standards for Drug Development and Approval. NeuroRx 2004;1:307-316.

5. Schneider LS. The potential and limits for clinical trials for early Alzheimer's disease and some recommendations. J Nutr Health Aging 2010;14:295-298

6. Sperling RA, Jack CR Jr, Aisen PS. Testing the right target and right drug at the right stage. Sci Transl Med 2011;3:111cm33.

7. Zahodne LB, Manly JJ, MacKay-Brandt A, Stern Y. Cognitive declines precede and predict functional declines in aging and Alzheimer's disease. PLoS One 2013;8:e73645.

8. Liu-Seifert H, Siemers E, Price K, et al. Cognitive impairment precedes and predicts functional impairment in mild Alzheimer's disease. J Alzheimers Dis 2015a;47:205-214

9. Grigsby J, Kaye K, Baxter J, Shetterly SM, Hamman RF. Executive cognitive abilities and functional status among community-dwelling older persons in the San Luis Valley Health and Aging Study. J Am Geriatrics Soc 1998;46:590596.
10. Cahn-Weiner DA, Malloy PF, Boyle PA, Marran M, Salloway S. Prediction of functional status from neuropsychological tests in community-dwelling elderly individuals. Clin Neuropsychol 2000;14:187-195

11. Royall DR, Palmer R, Chiodo LK, Polk MJ. Executive control mediates memory's association with change in instrumental activities of daily living: the Freedom House Study. J Am Geriatrics Soc 2005;53:11-17.

12. Cahn-Weiner DA, Ready RE, Malloy PF. Neuropsychological predictors of everyday memory and everyday functioning in patients with mild Alzheimer's disease. J Geriatr Psychiatry Neurol. 2003;16:84-89.

13. Boyle PA, Malloy PF, Salloway S, Cahn-Weiner DA, Cohen R, Cummings JL. Executive dysfunction and apathy predict functional impairment in Alzheimer disease. Am J Geriatr Psychiatry 2003;11:214-221.

14. Boyle PA, Paul RH, Moser DJ, Cohen RA. Executive impairments predict functional declines in vascular dementia. Clin Neuropsychol 2004;18:75-82.

15. Goldstein G, McCue M, Rogers J, Nussbaum PD. Diagnostic differences in memory test based predictions of functional capacity in the elderly. Neuropsychol Rehabil 992;2:307-317.

16. Farias ST, Harrell E, Neumann C, Houtz A. The relationship between neuropsychological performance and daily functioning in individuals with Alzheimer's disease: ecological validity of neuropsychological tests. Arch Clin Neuropsych 2003;18:655-672.

17. Jefferson AL, Byerly LK, Vanderhill S, et al. Characterization of activities of daily living in individuals with mild cognitive impairment. Am J Geriatr Psychiatry 2008;16:375-383.

18. Krall JR, Carlson MC, Fried LP, Xue QL. Examining the dynamic, bidirectional associations between cognitive and physical functioning in older adults. Am J Epidemiol 2014;180:838-846.

19. Liu-Seifert H, Siemers E, Sundell K, Price K, Han B, et al. Cognitive and functional decline and their relationship in patients with mild Alzheimer's dementia. J Alzheimers Dis 2015b;43:949-955.

20. [ADNI] Alzheimer's Disease Neuroimaging Initiative: Sharing Alzheimer's Research Data with the World, available at: adni.loni.usc.edu

21. Doody RS, Thomas RG, Farlow M, et al.; Alzheimer's Disease Cooperative Study Steering Committee; Solanezumab Study Group. Phase 3 trials of solanezumab for mild-to-moderate Alzheimer's disease. N Engl J Med 2014;370:311-321.

22. Doody RS, Raman R, Farlow M, et al. A Phase 3 Trial of Semagacestat for Treatment of Alzheimer's Disease. N Engl J Med 2013;369:341-350.

23. [ADNI] Alzheimer's Disease Neuroimaging Initiative, available at: www. adni-info.org.

24. Mohs RC, Knopman D, Petersen RC, et al. Development of cognitive instruments for use in clinical trials of antidementia drugs: additions to the Alzheimer's disease assessment scale that broaden its scope. Alzheimer Dis Assoc Disorder 1997;11:S13-21.

25. Galasko D, Kershaw PR, Schneider L, Zhu Y, Tariot PN. Galantamine maintains ability to perform activities of daily living in patients with Alzheimer's disease. J Am Geriatrics Soc 2004;52:1070-1076.

26. Pfeffer RI, Kurosaki TT, Harrah CH Jr, Chance JM, Filos S (1982) Measurement of functional activities in older adults in the community. J Gerontol 37, 323-329.

27. Vellas B, Andrieu S, Cantet C, Dartigues JF, Gauthier S. Long-term changes in ADAS-Cog: what is clinically relevant for disease modifying trials in Alzheimer?. J Nutr Health Aging. 2007;11:338-341.

28. Wajman JR, Oliveira FF, Marin SM, Schultz RR, Bertolucci PH. Is there correlation between cognition and functionality in severe dementia? the value of a performance-based ecological assessment for Alzheimer's disease. Arq Neuropsiquiatr 2014;72:845-850. 